\title{
BMJ Open Efficacy of a dual-ring wound protector for prevention of incisional surgical site infection after Whipple's procedure (pancreaticoduodenectomy) with preoperatively-placed intrabiliary stents: protocol for a randomised controlled trial
}

Alexsander K Bressan, ${ }^{1}$ Derek J Roberts, ${ }^{1,2}$ Janet P Edwards, ${ }^{1}$ Sana U Bhatti, ${ }^{1}$ Elijah Dixon, ${ }^{1}$ Francis R Sutherland, ${ }^{1}$ Oliver Bathe, ${ }^{1}$ Chad G Ball ${ }^{1}$

To cite: Bressan AK, Roberts DJ, Edwards JP, et al. Efficacy of a dual-ring wound protector for prevention of incisional surgical site infection after Whipple's procedure (pancreaticoduodenectomy) with preoperatively-placed intrabiliary stents: protocol for a randomised controlled trial. BMJ Open 2014;4: e005577. doi:10.1136/ bmjopen-2014-005577

- Prepublication history for this paper is available online. To view these files please visit the journal online (http://dx.doi.org/10.1136/ bmjopen-2014-005577).

Received 28 April 2014 Revised 28 July 2014 Accepted 1 August 2014

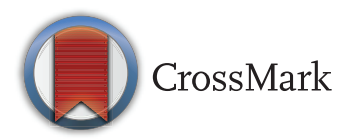

For numbered affiliations see end of article.

Correspondence to Dr Chad G Ball; Ball.Chad@gmail.com

\section{ABSTRACT}

Introduction: Among surgical oncology patients, incisional surgical site infection is associated with substantially increased morbidity, mortality and healthcare costs. Moreover, while adults undergoing pancreaticoduodenectomy with preoperative placement of an intrabiliary stent have a high risk of this type of infection, and wound protectors may significantly reduce its risk, no relevant studies of wound protectors yet exist involving this patient population. This study will evaluate the efficacy of a dual-ring wound protector for prevention of incisional surgical site infection among adults undergoing pancreaticoduodenectomy with preoperatively-placed intrabiliary stents.

Methods and analysis: This study will be a parallel, dual-arm, randomised controlled trial that will utilise a more explanatory than pragmatic attitude. All adults ( $\geq 18$ years) undergoing a pancreaticoduodenectomy at the Foothills Medical Centre in Calgary, Alberta, Canada with preoperative placement of an intrabiliary stent will be considered eligible. Exclusion criteria will include patient age $<18$ years and those receiving long-term glucocorticoids. The trial will employ block randomisation to allocate patients to a commercial dual-ring wound protector (the Alexis Wound Protector) or no wound protector and the current standard of care. The main outcome measure will be the rate of surgical site infection as defined by the Centers for Disease Control and Prevention criteria within 30 days of the index operation date as determined by a research assistant blinded to treatment allocation. Outcomes will be analysed by a statistician blinded to allocation status by calculating risk ratios and $95 \% \mathrm{Cls}$ and compared using Fisher's exact test. Ethics and dissemination: This will be the first randomised trial to evaluate the efficacy of a dual-ring wound protector for prevention of incisional surgical

\section{Strengths and limitations of this study}

- First randomised clinical trial to assess efficacy of a dual-ring wound protector in reducing the risk of incisional surgical site infection (SSI) after Whipple's procedure with preoperative placement of an intrabiliary stent.

- Incisional SSI will be diagnosed by systematic evaluation of surgical wounds using clear predefined criteria, within 30 days of surgery. Outcome assessor will be blinded to study allocation status of participants.

- This is a single centre study.

site infection among patients undergoing pancreaticoduodenectomy. Results of this study are expected to be available in 2016/2017 and will be disseminated using an integrated and end-of-grant knowledge translation strategy.

Trial registration number: ClinicalTrials.gov identifier NCT01836237.

\section{INTRODUCTION}

Surgical site infection (SSI) is a leading cause of preventable morbidity and mortality in North America and worldwide. This condition has consistently been reported to account for up to $25 \%$ of all healthcareassociated infections. ${ }^{1}{ }^{2}$ Prolonged hospitalisation, more frequent hospital re-admissions after surgery and a greater than twofold increase in costs and mortality have consistently been associated with this condition over the past decade. $^{3-9}$ As such, an 
increasing number of studies have cited an urgent need for safe and effective primary and secondary SSI prevention strategies, especially given the rising incidence of antimicrobial-resistant pathogens in hospital environments. ${ }^{10} 11$

As most cases of SSI are incisional, they are most often restricted to the skin or subcutaneous and deep soft tissues within the incision. ${ }^{12}$ These types of SSI are associated with an increased risk of postsurgical pain, poor wound healing and aesthetic results and an increased risk of incisional hernias. ${ }^{13}$ Antibiotic pharmacotherapy; need for intermittent dressing changes and/or wound debridement, prolonged hospitalisation and afterdischarge ambulatory or outpatient care; as well as lost personal work productivity and indirect medical costs also incur significant costs to the patient and healthcare system. $^{1415}$

In order to minimise the risk of SSI, several perioperative care interventions, including skin cleansing, hair removal, hypothermia prevention and perioperative antibiotic therapy, have been extensively studied over the past two decades. ${ }^{2}{ }^{16-20}$ However, few studies of the efficacy of surgical primary prevention manoeuvres aimed at reducing contamination within the surgical site exist, especially among patients undergoing prolonged and complex gastrointestinal operations. Thus, surgical procedures classified as contaminated or dirty (based on greater bacterial load within the surgical site and/or the elective or emergency opening of gastrointestinal or biliary tracts) remain associated with at least a twofold increased risk of wound infection as compared with clean and clean-contaminated surgeries. ${ }^{21}{ }^{22}$ Developing a more effective and reproducible primary prevention strategy for incisional SSIs among patients undergoing contaminated or dirty abdominal operations is therefore arguably a logical step towards further reducing the risk of SSI.

\section{Wound protectors}

The use of adhesive membrane barriers over the skin of the surgical site emerged 50 years ago as a possible solution to minimise endogenous cross-contamination during surgery. ${ }^{23}$ The initial idea relied on the principle of reducing exposure of the surgical site to bacteria inherent in the surrounding skin or to airborne bacteria in the operating room. Major applicability was expected in clean surgeries, where the skin is considered the main source of bacteria. Unfortunately, however, no evidence in support of plastic adhesive drapes was found in a recently updated systematic review of randomised controlled trials (RCTs) including five studies and 3082 participants. ${ }^{24}$ In fact, a $23 \%$ increase in the risk of SSI was found in the group that received adhesive drapes.

In the 1960s, other devices were described and then developed based on the concept of combining a nontraumatic surgical wound retractor with a protective membrane covering of the incisional margin in abdominal surgeries. Such protective covers or "wound protectors' were hypothesised to be an improvement over adhesive membrane barriers as they were believed to reduce intraoperative contamination while concomitantly preserving the temperature and humidity of the surgical wound. In support of this hypothesis, early studies demonstrated reduced exposure of the surgical wound to enteric bacteria at the end of gastrointestinal operations. ${ }^{25}{ }^{26}$ These results were further supported by several RCTs, which demonstrated that wound protectors were efficacious in reducing the incidence of incisional SSI as compared to usual care in patients undergoing gastrointestinal surgeries. ${ }^{27-29}$

A recently conducted meta-analysis of RCTs by our group, conducted in preparation for the trial described herein, evaluated whether diverse types of wound protectors may reduce the risk of SSI among patients undergoing gastrointestinal or biliary surgery. ${ }^{30}$ Among six trials, which enrolled 1008 patients, ${ }^{27} 2931-34$ we found that the pooled estimated risk of SSI among patients fitted with wound protectors during surgery was 0.55 (95\% CI 0.31 to 0.98 ) times the pooled estimated risk of SSI in control groups. Moreover, among patients treated with dual-ring versus single-ring wound protectors, the pooled estimated risk of SSI was 0.31 (95\% CI 0.14 to 0.67 ) times the pooled estimated risk in the control groups. As blinding status of outcome assessors was identified as a significant source of between-study heterogeneity, the design of the wound protector as well as the method of outcome classification may be important considerations for the design of future wound protector trials.

\section{SSI in Whipple's procedures with preoperatively-placed intrabiliary stents}

Over the past several decades, an increasing number of Whipple's procedures or pancreaticoduodenectomies have been performed. Major reasons include its expanding indications, an increasing ability to detect resectable pancreaticoduodenal lesions earlier in the course of the patient's disease, improvements in surgical technique and reduced perioperative mortality in high volume centres. ${ }^{35-40}$ However, despite a number of studies aimed at understanding whether technical factors contribute to outcome, ${ }^{41-43}$ postoperative morbidity remains high, ranging from $38 \%$ to $50 \%$, and mostly comprised of infectious complications. ${ }^{36} 4445$ Thus, newer studies have examined the importance of a broader approach that involves revising preoperative risk assessment, ${ }^{46}$ use of perioperative antibiotic prophylaxis, ${ }^{47-49}$ and refinement of the indications for preoperative biliary drainage.

Although preoperative biliary drainage was initially thought to lead to improved outcomes among patients awaiting surgery with bile duct obstruction (which frequently results from a regional mass effect secondary to the pancreaticobiliary pathology), this effect has not yet been demonstrated. ${ }^{50} 51$ As such, preoperative biliary drainage is now selectively used in some centres, especially among patients who are to receive neoadjuvant therapy, as well as for patients with jaundice complicated by cholangitis, impeding renal failure and other 
complications. ${ }^{52}$ Despite this, prolonged waiting time for pancreaticoduodenectomy, such as frequently occurs in ours and many other institutions, is a common and acceptable reason for use of preoperative biliary drainage, which aims to avoid the higher risk of complications associated with prolonged bile duct obstruction.

While the precise indications for preoperative biliary drainage are still undergoing debate, ${ }^{53}$ the association between preoperative biliary drainage, bile colonisation with bacteria and other microorganisms and an increased risk of infectious complications has repeatedly been demonstrated. In the early 2000s, three large cohort studies from tertiary care centres provided data supporting an increased risk of infectious complications after pancreaticoduodenectomy preceded by biliary drainage procedures. In one of these studies, the risk of infectious complications was $41 \%$ with preoperative biliary drainage versus $25 \%$ without preoperative biliary drainage among 240 consecutive patients who received a pancreaticoduodenectomy. ${ }^{44}$ Moreover, the odds of surgical wound infection has been reported to be 3.4 times higher among those that underwent pancreaticoduodenectomy and received preoperative biliary drainage as compared to those who did not. ${ }^{45}$ Thus, while preoperative biliary drainage may always be indicated for some patients, this treatment is likely associated with a high risk of associated SSIs.

\section{Objective}

Although incisional SSIs represent a common infectious complication following pancreaticoduodenectomy associated with preoperative use of intrabiliary stents, ${ }^{40} 54$ the efficacy and safety of wound protectors for reducing postoperative SSI has not yet been studied. Thus, the objective of this study is to evaluate the efficacy of a dual-ring wound protector versus no wound protector and the current standard of care for reducing the risk of 30-day incisional SSI among patients undergoing pancreaticoduodenectomy with preoperatively-placed intrabiliary stents.

\section{METHODS AND ANALYSIS Overview}

This study will be a single-centre, parallel-group, dual-arm RCT. Patients previously fitted with an intrabiliary drainage stent will be randomly assigned in the preoperative period to treatment with a commercial dual-ring wound protector (The Alexis Wound Protector (Applied Medical, Rancho Santa Margarita, California, USA)) or no wound protector and the current standard of care during the conduct of a pancreaticoduodenectomy (Whipple's procedure). As we hypothesise superiority of the dual-ring wound protector over the current standard of care, we plan to examine for evidence of this in statistical hypothesis tests. The CONSORT flow diagram $^{55}$ is presented in figure 1.

\section{Setting}

The study will take place at the Foothills Medical Centre (FMC), a University of Calgary-affiliated, tertiary care centre located in Calgary, Alberta, Canada. The FMC is
Figure 1 The trial CONSORT flow diagram.

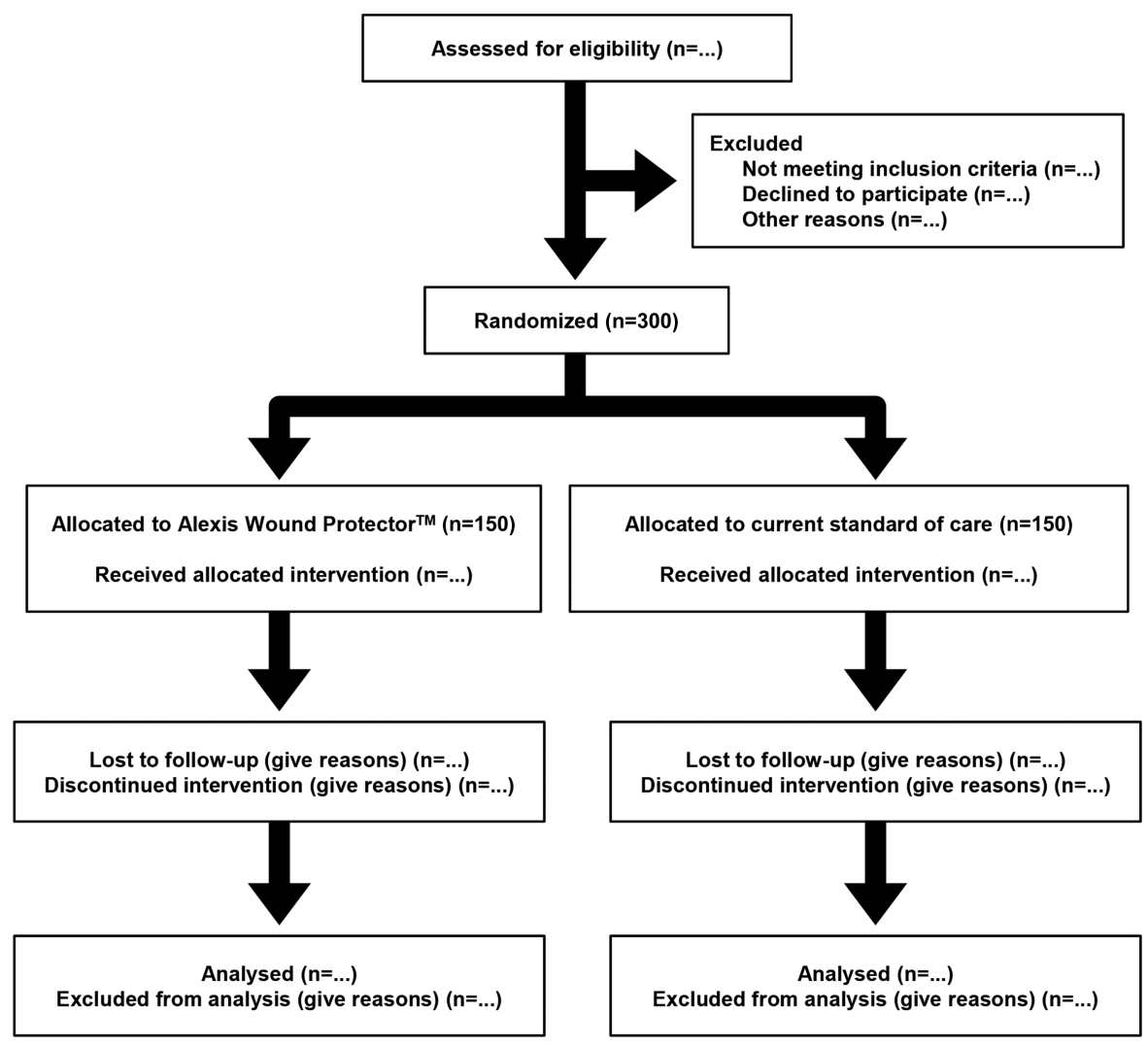


Figure 2 The trial Pragmatic-Explanatory Continuum Indicator Summary (PRECIS) Wheel. In this diagram, the encircled ' $E$ ' represents the extreme explanatory end of the pragmatic-explanatory continuum.

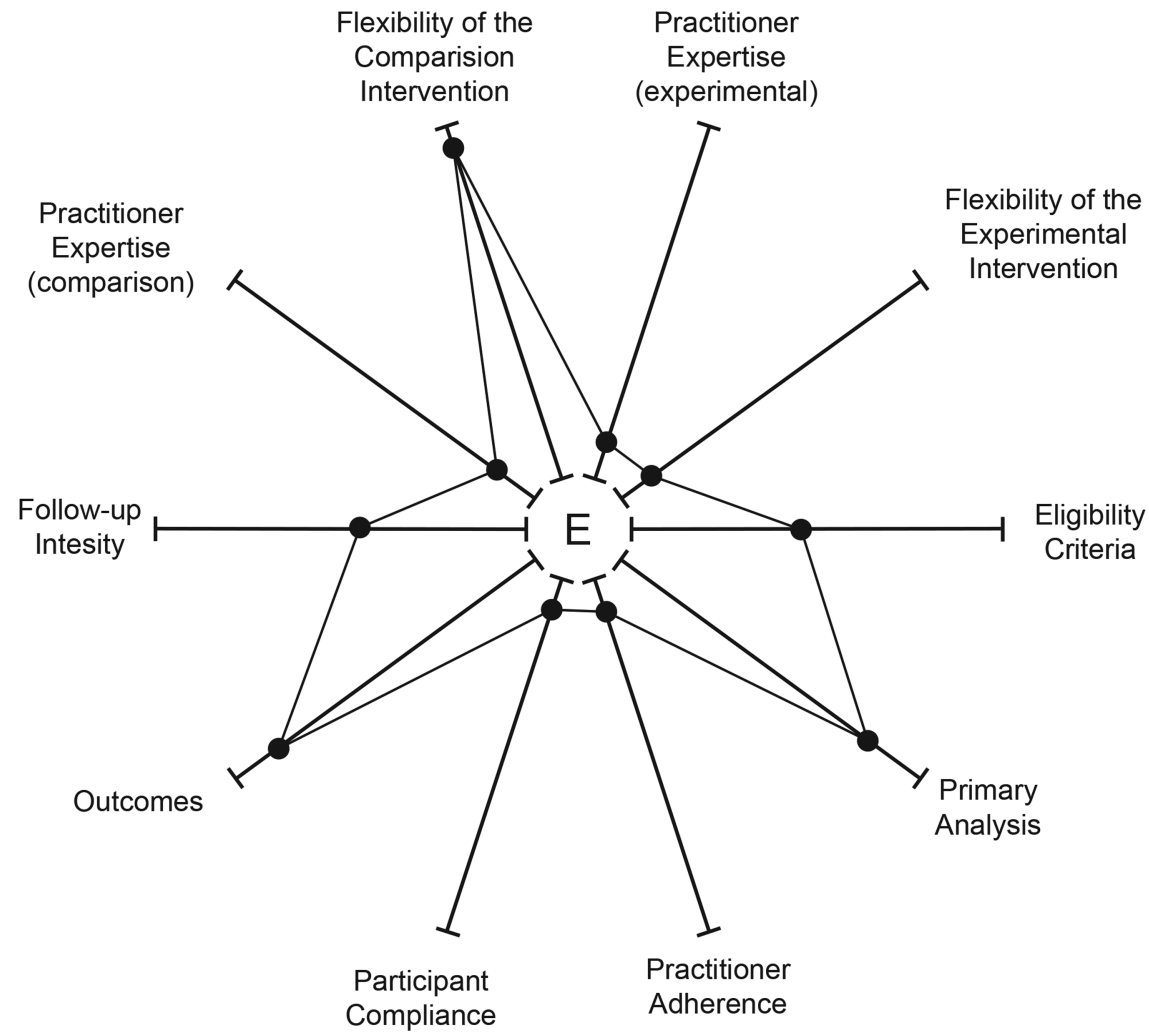

a high volume pancreaticoduodenectomy centre (approximately 125 procedures are performed in-house per year), which provides specialised surgical services for southern Alberta, southeast Saskatchewan and southwest British Columbia. The hospital offers a fellowship training programme in hepatobiliary surgery and a residency training programme in general surgery, and is staffed by four fellowship-trained hepatopancreatobiliary surgeons, all of which will participate in the study.

\section{Explanatory versus pragmatic trial attitude}

Although wound protectors have already been shown to be efficacious in preventing SSI in previous RCTs, they have not yet been demonstrated to be efficacious among patients undergoing pancreaticoduodenectomy with use of preoperatively-placed biliary stents. Thus, we will adopt a somewhat more explanatory than pragmatic trial attitude during trial design. ${ }^{56}$ Although specifics of the trial will be outlined below, the explanatory versus pragmatic nature is summarised visually through use of the PragmaticExplanatory Continuum Indicator Summary (PRECIS) Wheel in figure 2. ${ }^{5758}$

\section{Intervention choice rationale}

The Alexis Wound Protector was selected as the intervention of choice as this device is a dual-ring wound protector, and dual-ring wound protectors were found to be more efficacious than single-ring devices for prevention of postoperative SSI in a previous meta-analysis. ${ }^{30}$ Moreover, this device allows for tension adjustment of the membrane barrier over the incision margin, and its self-retaining design likely provides efficient retraction and circumferential sealing of the surgical wound during the conduct of abdominal surgery. The Alexis Wound Protector is composed of two rings, including the external white rigid retraction ring and the internal green flexible ring, which are interconnected through an impermeable transparent tubular membrane (figure 3). Six sizes are available for different lengths of surgical incision, which range from XX-small (for $1-3 \mathrm{~cm}$ incisions) to XX-large (for $17-25 \mathrm{~cm}$ incisions).

\section{Patient population and recruitment}

The study population will consist of all consecutive adult patients undergoing pancreaticoduodenectomy that had intrabiliary stents placed prior to the operation. Pancreaticoduodenectomy is indicated for patients with

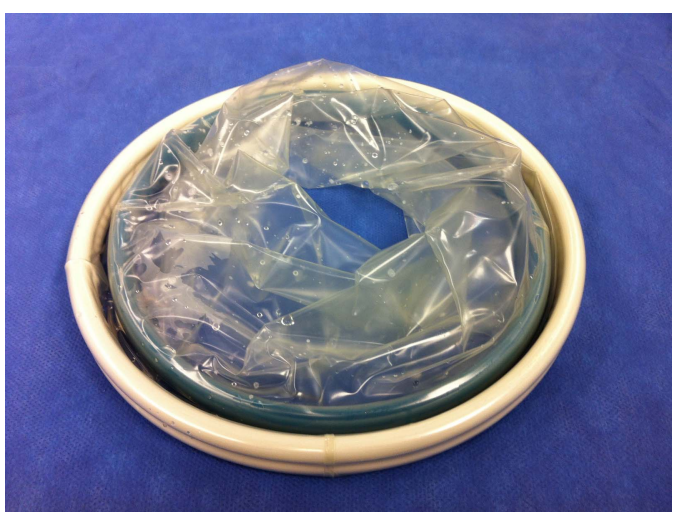

Figure 3 The Alexis Wound Protector ex vivo. 
periampullary tumours, deemed resectable on preoperative multiphasic computed tomography (CT) and/or magnetic resonance imaging (MRI). Borderline resectable pancreatic tumours are also considered for surgical treatment according to the National Comprehensive Cancer Network pancreatic cancer resectability criteria. ${ }^{59}$ Preoperative biliary drainage at our institution is not limited to patients with complicated obstructive jaundice, but rather represents the norm for patients with bile duct obstruction, given the typically prolonged surgical waiting time.

All consecutive patients who consent to undergo a pancreaticoduodenectomy at the FMC and who meet the inclusion/exclusion criteria will be contacted by a trained trial research assistant in order to obtain informed consent for enrolment in the trial. This research assistant will contact the patient prior to surgery by telephone and will discuss study procedures, risks and benefits and that enrolment in the trial is entirely voluntary. Among patients willing to participate in the trial, a consent form will subsequently be sent by mail in order to obtain written informed consent for randomisation and study enrolment.

The first participant was enrolled into the trial on April 26, 2013 and enrolment is currently ongoing.

Inclusion criteria

$\checkmark \geq 18$ years of age.

Exclusion criteria

- External biliary drainage.

- Long-term glucocorticoid use (as long-term use of these steroids, especially in high doses, is associated with increased risk of infections and preoperative use may predispose to development of incisional SSI) ${ }^{6061}$

- Patients unable to provide informed consent.

\section{Randomisation and blinding}

Variable block size randomisation with a 1:1 ratio will be performed by the research assistant using an online randomisation list generator website. In case of an odd number of participants, the last enrolled participant will be assigned to the following block. In order to maintain allocation concealment, the assigned intervention (ie, dual-ring wound protector or standard of care) will be provided to the surgeon in an opaque, sealed envelope immediately before surgery by the research assistant. Patients, outcome assessor, data analysts and charts will be blinded to treatment allocation status.

\section{Study intervention overview}

\section{Surgical conduct during and after pancreaticoduodenectomy}

The perioperative care provided to both intervention groups, including hair removal technique, skin preparation and antibiotic prophylaxis, will follow the routine practice of the surgical team and will not be standardised. Accordingly, surgical incision (midline supra-umbilical or bilateral subcostal incision), intraoperative use of sponges to contain contamination and saline irrigation of the surgical site at the end of the surgery will be employed according to the preference of the surgeon. This information will be recorded and reported in the manuscript.

Despite not being standardised, wound care in the trial will generally follow a relatively consistent process. This will include hair removal in the operating room with clippers followed by application of iodine or chlorhexidinebased antiseptic solutions. Cefazolin with metronidazole will be given for preoperative antibiotic prophylaxis before the skin incision is made. After the operative procedure, the abdominal wall will be closed with running sutures of looped 1 polydioxanone (PDS) in the muscular-aponeurotic plane (one layer for midline incision and two layers for bilateral subcostal), with staples placed in the skin.

\section{Application of the Alexis Wound Protector}

The Alexis Wound Protector will be applied immediately after achieving adequate access to the peritoneal cavity (figure 4). The size will be chosen by the surgeon according to Applied Medical instructions (large for 9-14 cm incisions, X-Large for $11-17 \mathrm{~cm}$ incisions and XX-Large for $17-25 \mathrm{~cm}$ incisions). Application and retrieval of the wound protector will also follow the manufacturer's instruction.

In brief, after achieving adequate access to the peritoneal cavity, the internal ring will be inserted through the incision, and placed in close apposition to the inner aspect of the incised abdominal wall. The external ring will then be rolled inward successive times until proper adjustment of tension and circumferential retraction of the plastic membrane over the incision margin. Gentle palpation around the internal ring will then be performed

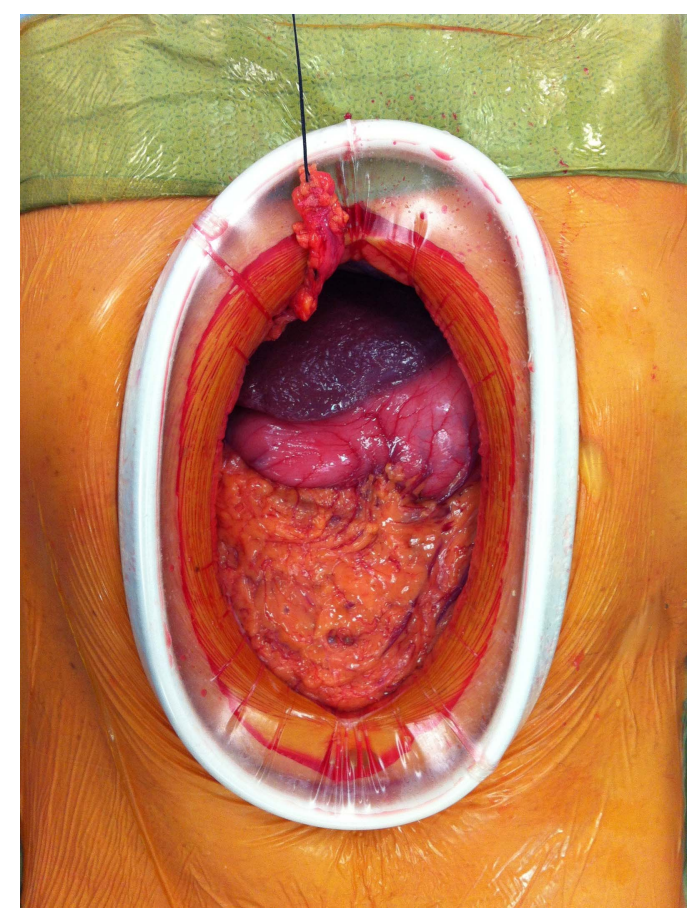

Figure 4 The Alexis Wound Protector fitted to the abdominal wall during the conduct of a pancreaticoduodenectomy. 
to confirm that no entrapment of intraperitoneal viscera has occurred in error. Removal of the wound protector will not occur until the end of the operation right before abdominal wall closure, during which the internal flexible ring will be carefully retrieved from the abdominal cavity.

\section{Study outcomes}

The primary outcome of the trial will be the risk of incisional SSI within 30 days of the operation.

Evaluation will consist of clinical examination of each patient's surgical wound on a daily basis during their in-hospital stay. After discharge, all patients will be referred to the outpatient surgery clinic for a follow-up assessment on the 30th postoperative day (or as close to this as is possible for the patient). Moreover, patients will also be informed to promptly contact the surgeon's office for an earlier consultation should they develop signs of incisional SSI (skin redness, pain or any drainage from the surgical wound). The date of postdischarge follow-up will be registered for all cases and reported in the results. The outcome assessor will be a research assistant trained by the surgical team to recognise the clinical manifestations of incisional SSI described below and who will be blinded to the treatment allocation status of the participants.

Incisional SSI will be diagnosed as superficial or deep according to the Centers for Disease Control and Prevention recommendations. ${ }^{62}$

\section{Superficial incisional SSI}

Involves only skin and subcutaneous tissue of the incision and the patient has at least one of the following:

1. Purulent drainage from the superficial incision.

2. Organisms isolated from an aseptically obtained culture of fluid or tissue from the superficial incision.

3. Superficial incision that is deliberately opened by a surgeon and is culture positive or not cultured, and the patient has at least one of the following signs or symptoms: pain or tenderness, localised swelling, redness or heat. However, a culture negative finding will not meet this criterion.

4. Diagnosis of superficial incisional SSI by the surgeon or attending physician.

\section{Deep incisional SSI}

Involves deep soft tissues of the incision and patient has at least one of the following:

1. Purulent drainage from the deep incision.

2. A deep incision that spontaneously dehisces or is deliberately opened by a surgeon and is culturepositive or not cultured, and patient has at least one of the following signs or symptoms: fever $\left(>38^{\circ} \mathrm{C}\right)$, localised pain or tenderness. A culture-negative finding does not meet this criterion.

3. An abscess or other evidence of infection involving the deep incision that is found on direct examination, during an invasive procedure or by histopathological examination or imaging test.
4. Diagnosis of a deep incisional SSI by a surgeon or attending physician.

\section{Study data collection}

Study data will be collected from the operative report and patient medical records by the research assistant. Data elements that will be collected include as below.

Patient demographics

- Date of birth

- Gender

- Preoperative use of chemotherapy

- Smoking status (including length of time that the patient has continuously been a smoker, and the number of cigarettes smoked per day on average)

- Comorbid conditions

- Previous abdominal surgeries

- Body mass index (weight in kilograms/height in metres squared)

- The American Society of Anesthesiologists' (ASA) classification for preoperative health assessment of surgical patients ${ }^{63}$

Perioperative care interventions

- Whether perioperative antibiotic prophylaxis was given, and the time of administration in relation to the beginning of the operative procedure

- Whether repeat doses of antibiotic prophylaxis were given (eg, as in prolonged operative procedures)

- Antibiotic type(s)

- Skin antiseptic type (ethanol-based, chlorhexidinebased or iodine-based)

- Whether hair was removed and the timing of its removal (prior to surgery or in the operating room)

Surgery characteristics/details

- Surgeon

- Whether a fellow, resident or medical student/clinical clerk was present

- Details of the surgical procedure

- Surgical wound classification (table 1$)^{64}$

- Use of surgical drains (and their type)

- Wound closure technique: suture type, size, layers (one or two), method (running or interrupted) and skin closure (staple or suture).

- Transfusion of blood products (packed red blood cells, platelets, fresh frozen plasma and albumin) and number of units

- Volume of intravenous crystalloid infusion

- Length of surgery (defined as the number of hours between skin incision and closure of skin)

\section{Data monitoring}

No data monitoring or interim analysis will be performed during the conduct of the trial.

\section{Sample size}

The sample size calculation is based on estimates obtained from a previous RCT comparing preoperative biliary drainage versus surgical management for cancer of the pancreatic head, ${ }^{65}$ and the estimated pooled relative risk of SSI 


\begin{tabular}{ll} 
Table 1 Classification of surgical wound & 64 \\
\hline Wound classification & Description \\
\hline Class I/clean & $\begin{array}{l}\text { An uninfected operative wound in which no inflammation is encountered and the respiratory, } \\
\text { alimentary, genital or uninfected urinary tract is not entered }\end{array}$ \\
$\begin{array}{l}\text { An operative wound in which the respiratory, alimentary, genital or urinary tracts are entered under } \\
\text { contaminated }\end{array}$ & $\begin{array}{l}\text { controlled conditions and without unusual contamination. Operations involving the biliary tract are } \\
\text { included in this category, provided no evidence of infection or major break in technique is } \\
\text { encountered } \\
\text { Open, fresh, accidental wounds. In addition, operations with major breaks in sterile technique (eg, } \\
\text { open cardiac massage) or gross spillage from the gastrointestinal tract and incisions in which } \\
\text { acute, nonpurulent inflammation is encountered are included in this category } \\
\text { Old traumatic wounds with retained devitalised tissue and those that involve existing clinical } \\
\text { infection or perforated viscera. This definition suggests that the organisms causing postoperative } \\
\text { infection were present in the operative field before the operation }\end{array}$ \\
\hline
\end{tabular}

reported by a previous systematic review and meta-analysis of the efficacy of dual-ring biliary stents. ${ }^{30}$ In order to achieve a power (superiority trial) of $80 \%$ and an $\alpha$ value of 0.05 , with a $13 \%$ expected incisional SSI rate in the control $^{65}$ versus $4 \%$ in the wound protector groups, ${ }^{30}$ the estimated total sample size required for the trial is $\mathbf{3 0 0}$ patients (150 in each arm).

Incisional SSI will be diagnosed by systematic evaluation of surgical wounds using clear predefined criteria, during 30 days after surgery. We therefore expect a more reliable, and higher, incidence of incisional SSI in our control group as compared to reports from previous retrospective studies. We also anticipate an accordingly greater absolute reduction in the incidence of incisional SSI in the wound protector group.

A too large sample size may have been initially calculated based on the limited and conservative parameters of incisional SSI incidence assumed for the study groups. Even so, our plan to finish recruitment and data collection in a 2-3-years period is justified by the high volume of Whipple's procedure at FMC, and the wide use of preoperative biliary stenting due to our long wait times for surgery. ${ }^{66}$

\section{Statistical analyses}

We will utilise an intention to treat analysis for the evaluation of all outcomes. All continuous variables will be summarised using histograms and measures of central tendency before statistical analyses are performed. Normal, continuous outcomes will be compared using t tests while non-normal, continuous outcomes will be compared using Mann-Whitney U tests. Categorical data will be summarised using proportions and by calculating risk ratios (RRs) with $95 \%$ CIs. These data will be compared using Fisher's exact test.

All tests will be two-sided, and those with a $\mathrm{p}$ value $<0.05$ will be considered statistically significant. Although the calculation of our primary outcome will be unadjusted for our primary analyses, if covariate imbalances occur between groups despite randomisation we will also adjust our RR estimates using a generalised linear model with a log link and binomial distribution (or a related model where necessary) as a sensitivity analysis. If this is performed, the unadjusted (primary) and adjusted (sensitivity) analyses will be reported in the manuscript. In addition to the calculation of the overall RR for SSI at 30 days, we will also conduct a subgroup analysis stratified by the type of operative procedure (palliative bypass vs curative resection). Stata/IC V.12.0 (Stata Corp. LP, College Station, Texas, USA) and R V.3.0.1 (available at http://www.r-project.org/) will be used for the conduct of all statistical analyses.

\section{Ethics and dissemination}

This will be the first RCT to assess whether a dual-ring wound protector is efficacious in reducing the risk of incisional SSI among adults undergoing Whipple's procedure preceded by preoperative placement of intrabiliary stents. As wound infection in this population is common and highly morbid, it may comprise a particularly suitable scenario for primary prevention through the use of abdominal wound protectors. Trial results are expected to be publicly available in $2016 / 2017$.

Our results will be disseminated to surgeons through an integrated and end-of-grant knowledge translation strategy. The International Hepato-Pancreato-Biliary Association, along with its Regional Associations and National Chapters represent a dynamic and consistent network integrating hepatobiliary surgeons across five continents. Two of our investigators (ED, CGB) are stakeholders in this organisation, and will be engaged throughout the conduct of the trial, from the setting of research questions through to the dissemination of results. After completion of the trial, results will be presented at major surgical and hepatobiliary surgery meetings.

\section{Author affiliations}

${ }^{1}$ Department of Surgery, University of Calgary and the Foothills Medical Centre, Calgary, Alberta, Canada

${ }^{2}$ Department of Community Health Sciences, Division of Epidemiology, University of Calgary, TRW (Teaching, Research, and Wellness), Calgary, Alberta, Canada 
Contributors CGB is the principal investigator and together with ED, FRS and $\mathrm{OB}$ has coordinated and actively participated in all the phases of trial design. These authors, as well as JPE, SUB and AKB assisted in drafting the protocol. CGB, ED, FRS and OB developed the ethics board application. JPE developed the data collection form. DJR, CGB and ED designed the statistical analysis plan. SUB and AKB assist in patient enrolment. AKB wrote the main manuscript. DJR critically revised the methodology and design of the trial protocol, as well as the writing of the manuscript. All authors contributed to the writing of the manuscript and agreed with submission of the final version for publication.

Funding Costs of the materials used in the study will be covered by a grant awarded by Applied Medical Resources Corporation (Rancho Santa Margarita, California, USA) to one of the trial investigators (CGB).

Competing interests DJR is supported by an Alberta Innovates-Health Solutions Clinician Fellowship Award, a Knowledge Translation Canada Strategic Training in Health Research Fellowship, and funding from the Canadian Institutes of Health Research and the Clinician Investigator Program at the University of Calgary.

Ethics approval Conjoint Health Research Ethics Board (CHREB) at the University of Calgary.

Provenance and peer review Not commissioned; externally peer reviewed.

Data sharing statement A final report including the final results from this planned study protocol is expected for publication in 2016/2017.

Open Access This is an Open Access article distributed in accordance with the Creative Commons Attribution Non Commercial (CC BY-NC 4.0) license, which permits others to distribute, remix, adapt, build upon this work noncommercially, and license their derivative works on different terms, provided the original work is properly cited and the use is non-commercial. See: http:// creativecommons.org/licenses/by-nc/4.0/

\section{REFERENCES}

1. Wenzel RP. Health care-associated infections: major issues in the early years of the 21st century. Clin Infect Dis 2007;45(Suppl 1): s85-88.

2. Al-Dabbagh MA, Dobson S. The evidence behind prophylaxis and treatment of wound infection after surgery. Adv Exp Med Biol 2013;764:141-50.

3. Broex EC, van Asselt AD, Bruggeman CA, et al. Surgical site infections: how high are the costs? J Hosp Infect 2009;72:193-201.

4. Kirkland KB, Briggs JP, Trivette SL, et al. The impact of surgical-site infections in the 1990s: attributable mortality, excess length of hospitalization, and extra costs. Infect Control Hosp Epidemiol 1999;20:725-30.

5. Carey K, Stefos T. Measuring the cost of hospital adverse patient safety events. Health Econ 2011;20:1417-30.

6. Carey K, Stefos T, Shibei Z, et al. Excess costs attributable to postoperative complications. Med Care Res Rev 2011;68:490-503.

7. Scott RD II Direct medical costs of healthcare-associated infections in U.S. hospitals and the benefits of prevention. http://www.cdc.gov/ hai/pdfs/hai/scott_costpaper.pdf

8. Kusachi S, Kashimura N, Konishi T, et al. Length of stay and cost for surgical site infection after abdominal and cardiac surgery in Japanese hospitals: multi-center surveillance. Surg Infect (Larchmt) 2012;13:257-65.

9. de Lissovoy G, Fraeman K, Hutchins V, et al. Surgical site infection: incidence and impact on hospital utilization and treatment costs. $\mathrm{Am}$ $J$ Infect Control 2009;37:387-97.

10. Allegranzi B, Bagheri Nejad S, Combescure C, et al. Burden of endemic health-care-associated infection in developing countries: systematic review and meta-analysis. Lancet 2011;377:228-41.

11. Rosenthal VD, Richtmann R, Singh S, et al. Surgical Site Infections, International Nosocomial Infection Control Consortium (INICC) Report, Data Summary of 30 Countries, 2005-2010. Infect Control Hosp Epidemiol 2013;34:597-604.

12. Horan TC, Gaynes RP, Martone WJ, et al. CDC definitions of nosocomial surgical site infections, 1992: a modification of CDC definitions of surgical wound infections. Infect Control Hosp Epidemiol 1992;13:606-8.

13. Le Huu Nho R, Mege D, Ouaïssi M, et al. Incidence and prevention of ventral incisional hernia. J Visc Surg 2012;149(Suppl 5):e3-14.
14. Alfonso JL, Pereperez SB, Canoves JM, et al. Are we really seeing the total costs of surgical site infections? A Spanish study. Wound Repair Regen 2007;15:474-81.

15. Perencevich EN, Sands KE, Cosgrove SE, et al. Health and economic impact of surgical site infections diagnosed after hospital discharge. Emerg Infect Dis 2003;9:196-203.

16. Owens CD, Stoessel K. Surgical site infections: epidemiology, microbiology and prevention. J Hosp Infect 2008;70(Suppl 2):3-10.

17. Swenson BR, Hedrick TL, Metzger R, et al. Effects of pre-operative skin preparation on postoperative wound infection rates: a prospective study of 3 skin preparation protocols. Infect control Hosp Epidemiol 2009:30:964-71.

18. Sebastian S. Does preoperative scalp shaving result in fewer postoperative wound infections when compared with no scalp shaving? A systematic review. J Neurosci Nurs 2012;44:149-56.

19. Gagliardi AR, Fenech D, Eskicioglu C, et al. Factors influencing antibiotic prophylaxis for surgical site infection prevention in general surgery: a review of the literature. Can J Surg 2009;52:481-9.

20. Berenguer CM, Ochsner MG Jr, Lord SA, et al. Improving surgical site infections: using national surgical quality improvement program data to institute surgical care improvement project protocols in improving surgical outcomes. J Am Coll Surg 2010;210:737-43.

21. Cruse P. Wound infection surveillance. Rev Infect Dis 1981;3:734-7.

22. Culver DH, Horan TC, Gaynes RP, et al. Surgical wound infection rates by wound class, operative procedure, and patient risk index. National Nosocomial Infections Surveillance System. Am J Med 1991;91:152s-7s.

23. Payne JT. An adhesive surgical drape. Am J Surg 1956;91:110-12.

24. Webster J, Alghamdi A. Use of plastic adhesive drapes during surgery for preventing surgical site infection. Cochrane Database Syst Rev 2013,1:CD006353.

25. Mohan HM, McDermott S, Fenelon L, et al. Plastic wound retractors as bacteriological barriers in gastrointestinal surgery: a prospective multi-institutional trial. J Hosp Infect 2012;81:109-13.

26. Horiuchi $\mathrm{T}$, Tanishima $\mathrm{H}$, Tamagawa $\mathrm{K}$, et al. A wound protector shields incision sites from bacterial invasion. Surg Infect (Larchmt) 2010;11:501-3.

27. Horiuchi T, Tanishima H, Tamagawa K, et al. Randomized, controlled investigation of the anti-infective properties of the Alexis retractor/protector of incision sites. J Trauma 2007;62:212-15.

28. Cheng KP, Roslani AC, Sehha N, et al. ALEXIS O-Ring wound retractor vs conventional wound protection for the prevention of surgical site infections in colorectal resections. Colorectal Dis 2012;14:e346-51.

29. Reid K, Pockney P, Draganic B, et al. Barrier wound protection decreases surgical site infection in open elective colorectal surgery: a randomized clinical trial. Dis Colon Rectum 2010:53:1374-80.

30. Edwards JP, Ho AL, Tee MC, et al. Wound protectors reduce surgical site infection: a meta-analysis of randomized controlled trials. Ann Surg 2012;256:53-9.

31. Lee P, Waxman K, Taylor B, et al. Use of wound-protection system and postoperative wound-infection rates in open appendectomy. Arch Surg 2009;144:872-5

32. Sookhai S, Redmond HP, Deasy JM. Impervious wound-edge protector to reduce postoperative wound infection: a randomised, controlled trial. Lancet 1999;353:1585

33. Gamble SS, Hopton DS. Plastic ring wound drapes in elective colorectal surgery. J R Coll Surg Edinb 1984;29:232-3.

34. Katthagen BD, Zamani P, Jung W. Effect of surgical draping on bacterial contamination in the surgical field. Z Orthop Ihre Grenzgeb 1992;130:230-5.

35. Teh SH, Diggs BS, Deveney CW, et al. Patient and hospital characteristics on the variance of perioperative outcomes for pancreatic resection in the United States: a plea for outcome-based and not volume-based referral guidelines. Arch Surg 2009;144:713-21.

36. Winter JM, Cameron JL, Campbell KA et al. 1423 pancreaticoduodenectomies for pancreatic cancer: a single-institution experience. J Gastrointest Surg 2006;10:1199-210.

37. Clancy TE, Ashley SW. Pancreaticoduodenectomy (Whipple operation). Surg Oncol Clin N Am 2005;14:533-52.

38. Fernandez-del Castillo C, Morales-Oyarvide V, McGrath D, et al. Evolution of the Whipple procedure at the Massachusetts General Hospital. Surgery 2012;152(3 Suppl 1):S56-63.

39. Ziegler KM, Nakeeb A, Pitt HA, et al. Pancreatic surgery: evolution at a high-volume center. Surgery 2010;148:702-9.

40. Ho V, Heslin MJ. Effect of hospital volume and experience on in-hospital mortality for pancreaticoduodenectomy. Ann Surg 2003;237:509-14

41. Patel AG, Toyama MT, Kusske AM, et al. Pylorus-preserving Whipple resection for pancreatic cancer: is it any better? Arch Surg 1995;130:838-42. 
42. Yeo CJ, Cameron JL, Maher MM, et al. A prospective randomized trial of pancreaticogastrostomy versus pancreaticojejunostomy after pancreaticoduodenectomy. Ann Surg 1995;222:580-8.

43. Bassi C, Falconi M, Molinari E, et al. Duct-to-mucosa versus end-to-side pancreaticojejunostomy reconstruction after pancreaticoduodenectomy: results of a prospective randomized trial. Surgery 2003;134:766-71.

44. Povoski SP, Karpeh MS Jr, Conlon KC, et al. Association of preoperative biliary drainage with postoperative outcome following pancreaticoduodenectomy. Ann Surg 1999;230:131-42.

45. Pisters PW, Hudec WA, Hess KR, et al. Effect of preoperative biliary decompression on pancreaticoduodenectomy-associated morbidity in 300 consecutive patients. Ann Surg 2001;234:47-55.

46. Parikh $\mathrm{P}$, Shiloach $\mathrm{M}$, Cohen $\mathrm{ME}$, et al. Pancreatectomy risk calculator: an ACS-NSQIP resource. HPB (Oxford) 2010; 12:488-97.

47. Donald GW, Sunjaya D, Lu X, et al. Perioperative antibiotics for surgical site infection in pancreaticoduodenectomy: Does the SCIP-approved regimen provide adequate coverage? Surgery 2013;154:190-6.

48. Kobayashi S, Gotohda N, Kato Y, et al. Infection control for prevention of pancreatic fistula after pancreaticoduodenectomy. Hepatogastroenterology 2013;60:876-82.

49. Lassen K, Coolsen MM, Slim K, et al. Guidelines for perioperative care for pancreaticoduodenectomy: Enhanced Recovery After Surgery (ERAS(R)) Society recommendations. World J Surg 2013;37:240-58.

50. Fang Y, Gurusamy KS, Wang Q, et al. Pre-operative biliary drainage for obstructive jaundice. Cochrane Database Syst Rev 2012;9:CD005444.

51. Nagino M, Takada T, Miyazaki M, et al. Preoperative biliary drainage for biliary tract and ampullary carcinomas. J Hepatobiliary Pancreat Surg 2008;15:25-30.

52. Rerknimitr R, Kullavanijaya P. Operable malignant jaundice: to stent or not to stent before the operation? World J Gastrointest Endosc 2010;2:10-14.

53. Iacono C, Ruzzenente A, Campagnaro T, et al. Role of preoperative biliary drainage in jaundiced patients who are candidates for pancreatoduodenectomy or hepatic resection: highlights and drawbacks. Ann Surg 2013;257:191-204.

54. Kent TS, Sachs TE, Callery MP, et al. The burden of infection for elective pancreatic resections. Surgery 2013;153:86-94.

55. Schulz KF, Altman DG, Moher D, CONSORT Group: CONSORT 2010 Statement: updated guidelines for reporting parallel group randomized trials. Trials 2010;11:32.

56. Schwartz D, Lellouch J. Explanatory and pragmatic attitudes in therapeutical trials. J Clin Epidemiol 2009;62:499-505.

57. Thorpe KE, Zwarenstein M, Oxman AD, et al. A pragmaticexplanatory continuum indicator summary (PRECIS): a tool to help trial designers. J Clin Epidemiol 2009;13:464-75.

58. Thorpe KE, Zwarenstein M, Oxman AD, et al. A pragmatic-explanatory continuum indicator summary (PRECIS): a tool to help trial designers. CMAJ 2009;180:E47-57.

59. Callery MP, Chang KJ, Fishman EK, et al. Pretreatment assessment of resectable and borderline resectable pancreatic cancer: expert consensus statement. Ann Surg Oncol 2009;16:1727-33.

60. Gil-Egea MJ, Pi-Sunyer MT, Verdaguer A, et al. Surgical wound infections: prospective study of 4,486 clean wounds. Infect Control 1987;8:277-80.

61. Slaughter MS, Olson MM, Lee JT Jr, et al. A fifteen-year wound surveillance study after coronary artery bypass. Ann Thorac Surg 1993;56:1063-8.

62. Centers for Disease Control and Prevention: CDC/NHSN Protocol corrections, clarification, and additions. April 2013. http://www.cdc. gov/nhsn/pdfs/pscmanual/9pscssicurrent.pdf

63. Dripps RD. New classification of physical status. Anesthesiol 1963;24:111.

64. Simmons BP. Guideline for prevention of surgical wound infections. Infect Control 1982;3:185-96.

65. van der Gaag NA, Rauws EA, van Eijck $\mathrm{CH}$, et al. Preoperative biliary drainage for cancer of the head of the pancreas. $N$ Engl $J$ Med 2010;362:129-37.

66. McLean SR, Karsanji D, Wilson J, et al. The effect of wait times on oncological outcomes from periampullary adenocarcinomas. J Surg Onc 2013;107:853-8. 